TRANSACTIONS OF THE

AMERICAN MATHEMATICAL SOCIETY

Volume 350, Number 7, July 1998, Pages 2833-2846

S $0002-9947(98) 02267-3$

\title{
ON THE AVERAGES OF DARBOUX FUNCTIONS
}

\author{
ALEKSANDER MALISZEWSKI
}

\begin{abstract}
Let $\mathbf{A}$ be the family of functions which can be written as the average of two comparable Darboux functions. In 1974 A. M. Bruckner, J. G. Ceder, and T. L. Pearson characterized the family $\mathbf{A}$ and showed that if $\alpha \geq 2$, then $\mathbf{A} \cap \mathbf{B}_{\alpha}$ is the family of the averages of comparable Darboux functions in Baire class $\alpha$. They also asked whether the latter result holds true also for $\alpha=1$. The main goal of this paper is to answer this question in the negative and to characterize the family of the averages of comparable Darboux Baire one functions.
\end{abstract}

\section{Preliminaries}

The letters $\mathbb{R}, \mathbb{Z}$, and $\mathbb{N}$ denote the real line, the set of integers, and the set of positive integers, respectively. We consider cardinals as ordinals not in one-to-one correspondence with the smaller ordinals. The word interval means a nondegenerate interval. The word function denotes a mapping from $\mathbb{R}$ into $\mathbb{R}$ unless otherwise explicitly stated. For each $A \subset \mathbb{R}$ we use the symbols $\operatorname{int} A, \operatorname{cl} A, \operatorname{fr} A, \chi_{A}$, and $|A|$ to denote the interior, the closure, the boundary, the characteristic function, and the cardinality of $A$, respectively. We write $\mathfrak{c}=|\mathbb{R}|$ and use the symbol $\operatorname{cf}(\mathfrak{c})$ to denote the cofinality of $\mathfrak{c}$. By $\mathfrak{a}_{m}$ and $\mathfrak{a}_{c}$ we denote the additivity of the $\sigma$-ideal of null sets and the additivity of the $\sigma$-ideal of meager sets, respectively.

Let $A \subset \mathbb{R}$ and $f: A \rightarrow \mathbb{R}$. For every $y \in \mathbb{R}$ let $[f<y]=\{x \in A: f(x)<y\}$. Similarly we define the symbols $[f \leq y],[f>y]$, etc. If $B \subset A$ and $|B|=\mathfrak{c}$, then let $\mathfrak{c}-\inf (f, B)=\inf \{y \in \mathbb{R}:|[f<y] \cap B|=\mathfrak{c}\}$ and $\mathfrak{c}-\sup (f, B)=-\mathfrak{c}-\inf (-f, B)$. If $x$ is a left $\mathfrak{c}$-limit point of $A$ (i.e., $|A \cap(x-\delta, x)|=\mathfrak{c}$ for every $\delta>0)$, then we denote $\mathfrak{c}-\underline{\lim }\left(f, x^{-}\right)=\lim _{\delta \rightarrow 0^{+}} \mathfrak{c}-\inf (f, A \cap(x-\delta, x))$ and $\mathfrak{c}-\overline{\lim }\left(f, x^{-}\right)=-\mathfrak{c}-\underline{\lim }\left(-f, x^{-}\right)$. Similarly we define the symbols $\mathfrak{c}-\lim \left(f, x^{+}\right)$and $\mathfrak{c}-\overline{l i m}\left(f, x^{+}\right)$if $x$ is a right $\mathfrak{c}$-limit point of $A$. If $B \subset A$ is nonempty, then let $\omega(f, B)$ be the oscillation of $f$ on $B$, i.e., $\omega(f, B)=\sup \{|f(x)-f(t)|: x, t \in B\}$. For each $x \in A$ let $\omega(f, x)$ be the oscillation of $f$ at $x$, i.e., $\omega(f, x)=\lim _{\delta \rightarrow 0^{+}} \omega(f, A \cap(x-\delta, x+\delta))$. The symbol $\mathrm{C}_{f}$ denotes the set of points of continuity of $f$. Thus $\mathcal{C}_{f}=\{x \in A: \omega(f, x)=0\}$. Let $\|f\|=\sup \{|f(x)|: x \in A\}$. Finally let $\mathcal{B}_{f}$ consist of all $x \in A$ such that $x$ is not a bilateral c-limit point of $A$ or $\max \left\{\mathfrak{c}-\underline{\lim }\left(|f-f(x)|, x^{-}\right), \mathfrak{c}-\underline{\lim }\left(|f-f(x)|, x^{+}\right)\right\}>0$.

The following denote classes of functions.

Received by the editors July 30, 1996.

1991 Mathematics Subject Classification. Primary 26A21, 54C30. Secondary 26A15, 54C08.

Key words and phrases. Darboux function, comparable functions, average of functions.

Partially supported by NSF Cooperative Research Grant INT-9600548, with its Polish part being financed by the Polish Academy of Sciences. 
A consists of all functions which can be written as the average of two comparable ${ }^{1}$ Darboux functions.

$\mathcal{B}$ consists of all functions possessing the Baire property.

$\mathbf{B}_{\alpha}$ denotes Baire class $\alpha\left(1 \leq \alpha<\omega_{1}\right)$.

$\mathbf{B}_{1}^{*}$ consists of all Baire one star functions [16], i.e., $f \in \mathbf{B}_{1}^{*}$ iff for each nonempty perfect set $P \subset \mathbb{R}$ there is an open interval $I$ such that $P \cap I \neq \emptyset$ and $f \uparrow(P \cap I)$ is continuous.

$\mathbf{C}_{q}$ consists of all cliquish functions, i.e., $f \in \mathbf{C}_{q}$ iff $\operatorname{cl} \mathfrak{C}_{f}=\mathbb{R}$. Cliquish functions are also called pointwise discontinuous. Clearly $\mathbf{B}_{1} \subset \mathbf{C}_{q} \subset \mathcal{B}$.

$\mathbf{D}$ consists of all Darboux functions, i.e., $f \in \mathbf{D}$ iff the set $f[J]$ is connected for every interval $J$.

L consists of all Lebesgue measurable functions.

We omit the intersection sign between classes of functions, e.g., $\mathbf{D B}_{1}$ is the family of all Darboux Baire one functions.

\section{INTRODUCTION}

In 1974 A. M. Bruckner, J. G. Ceder, and T. L. Pearson proved that a function, $f$, can be written as the average of two comparable Darboux functions $\varphi$ and $\psi$ if and only if for each $x \in \mathbb{R}$ we have $\max \left\{\mathfrak{c}-\underline{\lim }\left(f, x^{-}\right), \mathfrak{c}-\underline{\lim }\left(f, x^{+}\right)\right\}<\infty$ and $\min \left\{\mathfrak{c}-\overline{\lim }\left(f, x^{-}\right), \mathfrak{c}-\varlimsup \overline{l i m}\left(f, x^{+}\right)\right\}>-\infty[4$, Theorem 2]. Moreover they showed we can conclude that $\varphi, \psi \in \mathbf{L}$ provided $f \in \mathbf{L}$, and that $\varphi, \psi \in \mathbf{B}_{\alpha}(\alpha \geq 2)$ provided $f \in \mathbf{B}_{\alpha}$. They also asked whether the latter result holds true also for $\alpha=1$ $[4$, p. 986]. The problem whether this assertion is true even for bounded Baire one functions was restated in 1983 by J. G. Ceder and T. L. Pearson [8, p. 186]. ${ }^{2}$

A similar problem to that considered by A. M. Bruckner, J. G. Ceder, and T. L. Pearson is to determine a necessary and sufficient condition that for a function $f$ there exists a Darboux function $\psi$ such that $\psi>f$ on $\mathbb{R}$. (The answer to this question in general case can be easily obtained using the proof of [4, Theorem 2].) In both cases we ask whether there is a positive function, $g$, such that both $f+g$ and $-f+g$ are Darboux (the first problem) or such that $f+g$ is Darboux (the second problem). It suggests a similar problem for larger classes of functions, even families of cardinality $\mathfrak{c}$ or greater. Theorem 4.1 contains a solution of this problem for classes of functions of cardinality less than or equal to $\mathfrak{c}$. It is an open problem whether it is consistent with $Z F C$ that condition (ii) of this theorem is sufficient for families of functions of cardinality $\mathfrak{c}^{+}$. (Cf. [9].)

Theorems 4.2 and 4.6 are similar to [11, Theorem 6]. Recall that by [11, Example 1], condition (ii) of Theorem 4.2 may not be sufficient if $|\mathfrak{A}| \geq \operatorname{cf}(\mathfrak{c})$. (See Remark 4.1.) However, I do not know whether the assumptions $|\mathfrak{A}|<\mathfrak{a}_{m}$ and $|\mathfrak{A}|<\mathfrak{a}_{c}$ are essential in Theorem 4.6. I also do not know whether we may allow uncountable families in Theorem 4.7. (See Problem 7.1.) By [11, Example 2], we cannot allow infinite families in Theorem 5.2. We will show that, unlike [10, Theorem 2] and [12, Theorem 4], we cannot conclude in condition (ii) of Theorem 5.2 that $g \in \mathbf{B}_{1}$. Actually, we cannot even conclude that $g$ is Borel measurable. (See Corollary 5.5.)

\footnotetext{
${ }^{1}$ We say that functions $\varphi$ and $\psi$ are comparable if either $\varphi<\psi$ on $\mathbb{R}$ or $\varphi>\psi$ on $\mathbb{R}$.

${ }^{2}$ The answer to this question follows immediately from [11, Theorem 8]. Indeed, let $f \in \mathbf{B}_{1}$ be bounded. By [11, Theorem 8], there are bounded functions $\varphi, \psi \in \mathbf{D B}_{1}$ such that $\varphi+\psi=2 f$. Since $\varphi$ and $\psi$ are bounded, we may assume that $\varphi<0<\psi$ on $\mathbb{R}$.
} 
As usual, the Baire class one case is solved in a separate theorem (Theorem 6.9). Notice that by Proposition 6.10, the necessary and sufficient condition for functions in Baire class one is essentially stronger than in the general case.

Corollaries 4.3, 4.4, and 4.5 are formulated only for the general case. (In particular, Corollary 4.5 is a generalization of [4, Theorem 2].) However, one can easily prove analogous results for Darboux Lebesgue measurable functions, Darboux Borel measurable functions, etc.

\section{Auxiliary Results}

The proof of the next lemma is straightforward. (See also [11, Lemma 5].)

Lemma 3.1. Suppose $\kappa \leq \mathfrak{c}$ and let $\left\{P_{\alpha}: \alpha<\kappa\right\}$ be a sequence of subsets of $\mathbb{R}$ of cardinality $\mathfrak{c}$. There is a sequence $\left\{Q_{\alpha}: \alpha<\kappa\right\}$ of pairwise disjoint sets of cardinality $\mathfrak{c}$ such that $Q_{\alpha} \subset P_{\alpha}$ for each $\alpha$. If each $P_{\alpha}$ is Lebesgue measurable (has the Baire property), then we can conclude that each $Q_{\alpha}$ is a null set (a nowhere dense set, respectively).

A similar result has been proved by J. Smítal [18, Lemma 2].

Lemma 3.2. Let $\left\{P_{n}: n \in \mathbb{N}\right\}$ be a sequence consisting of uncountable Borel measurable sets. There exists a sequence, $\left\{Q_{n}: n \in \mathbb{N}\right\}$, consisting of pairwise disjoint nonempty null perfect sets, such that $Q_{n} \subset P_{n}$ for each $n$.

The next lemma follows immediately from the definition.

Lemma 3.3. Let $B, A_{1}, A_{2}, \ldots \subset \mathbb{R}$ be such that $\left|B \cap A_{n}\right|=\mathfrak{c}$ for each $n \in \mathbb{N}$ and $\left|B \backslash \bigcup_{n \in \mathbb{N}} A_{n}\right|<\mathfrak{c}$. Then for every function $f: B \rightarrow \mathbb{R}$ we have

$$
\mathfrak{c}-\sup (f, B)=\sup \left\{\mathfrak{c}-\sup \left(f, B \cap A_{n}\right): n \in \mathbb{N}\right\} .
$$

Lemma 3.4. Let $A \subset \mathbb{R}$ and $f: A \rightarrow \mathbb{R}$. Then $\left|\mathcal{B}_{f}\right|<\mathfrak{c}$. If $A$ and $f$ are Lebesgue measurable ( $A$ and $f$ have the Baire property, $A$ and $f$ are Borel measurable), then moreover $\mathcal{B}_{f}$ is a null set $\left(\mathcal{B}_{f}\right.$ is a meager set, $\mathcal{B}_{f}$ is at most countable, respectively).

Proof. The main assertion follows by [7, Lemma 4, p. 285]. (See also [6, Lemma 1].) The additional assertions can be proved in essentially the same manner, using the fact that for each $A \subset \mathbb{R}$, if $|A|<\mathfrak{c}$ and $A$ is Lebesgue measurable ( $A$ has the Baire property, $A$ is Borel measurable), then $A$ is a null set ( $A$ is a meager set, $A$ is at most countable, respectively).

The next result is due to I. Maximoff [13].

Lemma 3.5. For each Borel measurable function $f$ and each nonempty perfect set $P$ we can find a countable set $B$ with the property that for each $x \in P \backslash B$ there is a perfect set $Q \subset P$ having $x$ as a bilateral limit point such that $f \backslash Q$ is continuous.

In 1965 A. M. Bruckner and J. L. Leonard showed that for each nonempty perfect set $P \subset \mathbb{R}$ there is a non-constant Darboux lower semicontinuous function which vanishes outside of $P$ [5, Corollary, p. 936]. Using this result, it is easy to prove the following lemmas.

Lemma 3.6. Let $P \subset \mathbb{R}$ be a nonempty perfect set. There is a Darboux upper semicontinuous function $g$ such that $g=0$ outside of $P$ and $g[P]=[0,1)$.

Lemma 3.7. Let $Q \subset \mathbb{R}$ be a nonempty perfect set. For each $y \in \mathbb{R}$ there is a Baire one function $\varphi$ such that $\varphi[Q]=(y, \infty)$. 


\section{General CASE}

Theorem 4.1. Let $\kappa \leq \mathfrak{c}$ and let $\mathfrak{A}=\left\{f_{\alpha}: \alpha<\kappa\right\}$ be a family of functions. The following are equivalent:

(i) there is a positive function $g$ such that $f_{\alpha}+g \in \mathbf{D}$ for each $\alpha<\kappa$;

(ii) there is a nonnegative function $h$ such that for each $\alpha<\kappa$ and each $x \in \mathbb{R}$ we have $\max \left\{\mathfrak{c}-\underline{\lim }\left(f_{\alpha}+h, x^{-}\right), \mathfrak{c}-\underline{\lim }\left(f_{\alpha}+h, x^{+}\right)\right\} \leq\left(f_{\alpha}+h\right)(x)$.

Proof. The implication (i) $\Rightarrow$ (ii) is evident. To prove the opposite implication, arrange all open intervals with rational end points in a sequence, $\left\{I_{n}: n \in \mathbb{N}\right\}$. For each $\alpha<\kappa$ and each $n \in \mathbb{N}$ set $y_{\alpha n}=\max \left\{\inf \left(f_{\alpha}+h\right)\left[I_{n}\right]+n^{-1},-n\right\}$, and define $K_{\alpha n}=\left[f_{\alpha}+h<y_{\alpha n}\right] \cap I_{n}$. By assumption, $\left|K_{\alpha n}\right|=\mathfrak{c}$ for each $\alpha$ and $n$. Use Lemma 3.1 to construct a family, $\left\{Q_{\alpha n}: \alpha<\kappa, n \in \mathbb{N}\right\}$, consisting of pairwise disjoint sets of cardinality $\mathfrak{c}$, such that each $Q_{\alpha n}$ is a subset of $K_{\alpha n}$. For each $\alpha$ and $n$ let $g_{\alpha n}: Q_{\alpha n} \rightarrow\left(y_{\alpha n}, \infty\right)$ be a surjection. Define $g(x)=g_{\alpha n}(x)-f_{\alpha}(x)+1$ if $x \in Q_{\alpha n}$ for some $\alpha<\kappa$ and $n \in \mathbb{N}$, and $g(x)=h(x)+1$ otherwise.

Clearly $g \geq h+1$ on $\mathbb{R}$, so $g$ is positive. Fix an $\alpha<\kappa$ and let $J$ be an interval. By assumption and Lemma 3.3, we obtain

$$
\inf \left(f_{\alpha}+h\right)[J] \geq \mathfrak{c}-\inf \left(f_{\alpha}+h, J\right)=\inf \left\{\mathfrak{c}-\inf \left(f_{\alpha}+h, I_{n}\right): I_{n} \subset J\right\} .
$$

Hence $\left(\inf \left(f_{\alpha}+h\right)[J]+1, \infty\right) \subset\left(f_{\alpha}+g\right)[J] \subset\left[\inf \left(f_{\alpha}+h\right)[J]+1, \infty\right)$. It follows that $\left(f_{\alpha}+g\right)[J]$ is an interval and $f_{\alpha}+g \in \mathbf{D}$.

Theorem 4.2. Let $\mathfrak{A}$ be a family of functions with $|\mathfrak{A}|<\operatorname{cf}(\mathfrak{c})$. The following are equivalent:

(i) there is a positive function $g$ such that $f+g \in \mathbf{D}$ for each $f \in \mathfrak{A}$;

(ii) for each $x \in \mathbb{R}$ we have

$$
\sup \left\{\max \left\{\mathfrak{c}-\underline{\lim }\left(f, x^{-}\right), \mathfrak{c}-\underline{\lim }\left(f, x^{+}\right)\right\}-f(x): f \in \mathfrak{A}\right\}<\infty .
$$

Proof. (i) $\Rightarrow$ (ii). For each $x \in \mathbb{R}$ and each $f \in \mathfrak{A}$, since $g$ is positive and $f+g \in \mathbf{D}$,

$$
\begin{aligned}
& \max \left\{\mathfrak{c}-\underline{\lim }\left(f, x^{-}\right), \mathfrak{c}-\underline{\lim }\left(f, x^{+}\right)\right\} \\
& \leq \max \left\{\mathfrak{c}-\underline{\lim }\left(f+g, x^{-}\right), \mathfrak{c}-\underline{\lim }\left(f+g, x^{+}\right)\right\} \leq(f+g)(x) .
\end{aligned}
$$

Thus for each $x \in \mathbb{R}$ the left-hand side of the inequality $(\star)$ does not exceed $g(x)$.

(ii) $\Rightarrow$ (i). We will show that condition (ii) of Theorem 4.1 holds. Let $h(x)$ equal the left-hand side of the inequality $(\star)$ if it is nonnegative, and zero otherwise. Then $h$ is finite and nonnegative. By Lemma 3.4 and our assumption, we get $|A|<\mathfrak{c}$, where $A=\bigcup_{f \in \mathfrak{A}} \mathcal{B}_{f}$. Notice that $h=0$ outside of $A$, so for each $f \in \mathfrak{A}$ and each $x \in \mathbb{R}$ we have

$$
\begin{aligned}
\max \left\{\mathfrak{c}-\underline{\lim }\left(f+h, x^{-}\right), \mathfrak{c}-\underline{\lim }\right. & \left.\left(f+h, x^{+}\right)\right\} \\
= & \max \left\{\mathfrak{c}-\underline{\lim }\left(f, x^{-}\right), \mathfrak{c}-\underline{\lim }\left(f, x^{+}\right)\right\} \leq(f+h)(x) .
\end{aligned}
$$

Remark 4.1. By [11, Example 1], there exists a family of functions, $\mathfrak{A}$, such that $|\mathfrak{A}|=\operatorname{cf}(\mathfrak{c}),\|f\| \leq 1$ for each $f \in \mathfrak{A}$ (so, in particular, the inequality ( $\star$ ) holds for each $x \in \mathbb{R}$ ) but for each nonnegative function $g$ there is an $f \in \mathfrak{A}$ with $f+g \notin \mathbf{D}$.

Corollaries 4.3 and 4.4 follow directly from Theorem 4.2.

Corollary 4.3. Let $\mathfrak{A}$ be a finite family of functions. The following are equivalent:

(i) there is a positive function $g$ such that $f+g \in \mathbf{D}$ for each $f \in \mathfrak{A}$; 
(ii) the inequality $\max \left\{\mathfrak{c}-\underline{\lim }\left(f, x^{-}\right), \mathfrak{c}-\underline{\lim }\left(f, x^{+}\right)\right\}<\infty$ holds for every $f \in \mathfrak{A}$ and every $x \in \mathbb{R}$.

Corollary 4.4. For each function $f$ the following are equivalent:

(i) there is a function $\psi \in \mathbf{D}$ such that $\psi>f$ on $\mathbb{R}$;

(ii) there is a function $\psi \in \mathbf{D}$ such that $\psi>f$ on $\mathbb{R}$ and $\psi-f \in \mathbf{D}$;

(iii) for every $x \in \mathbb{R}$ we have $\max \left\{\mathfrak{c}-\underline{\lim }\left(f, x^{-}\right), \mathfrak{c}-\underline{\lim }\left(f, x^{+}\right)\right\}<\infty$.

Corollary 4.5. For each function $f$ the following are equivalent:

(i) there are functions $\varphi, \psi \in \mathbf{D}$ such that $\varphi<f<\psi$ on $\mathbb{R}$;

(ii) there are functions $\varphi, \psi \in \mathbf{D}$ such that $\varphi<f<\psi$ and $f=(\varphi+\psi) / 2$ on $\mathbb{R}$, and $f-\varphi=\psi-f \in \mathbf{D}$

(iii) for every $x \in \mathbb{R}$ we have both $\max \left\{\mathfrak{c}-\underline{\lim }\left(f, x^{-}\right), \mathfrak{c}-\underline{\lim }\left(f, x^{+}\right)\right\}<\infty$ and $\min \left\{\mathfrak{c}-\overline{l i m}\left(f, x^{-}\right), \mathfrak{c}-\overline{\lim }\left(f, x^{+}\right)\right\}>-\infty$.

Proof. The implication (ii) $\Rightarrow$ (i) is evident. The other two implications follow easily by Corollary 4.3 if we let $\mathfrak{A}=\{f,-f, 0\}$, and put $\varphi=f-g$ and $\psi=f+g$.

Theorem 4.6. For each family $\mathfrak{A} \subset \mathbf{L}$ (respectively for each family $\mathfrak{A} \subset \mathcal{B})$, if $|\mathfrak{A}|<\mathfrak{a}_{m}$ (respectively $\left.|\mathfrak{A}|<\mathfrak{a}_{c}\right)$, then the following are equivalent:

(i) there is a positive function $g$ such that $f+g \in \mathbf{D}$ for each $f \in \mathfrak{A}$ and $[g \neq 0]$ is a null set (a meager set, respectively);

(ii) there is a nonnegative function $h$ such that for each $f \in \mathfrak{A}$ and $x \in \mathbb{R}$ we have $\max \left\{\mathfrak{c}-\underline{\lim }\left(f+h, x^{-}\right), \mathfrak{c}-\underline{\lim }\left(f+h, x^{+}\right)\right\} \leq(f+h)(x)$

(iii) the inequality $(\star)$ holds for each $x \in \mathbb{R}$.

Proof. The proof mimics the arguments used in Theorems 4.1 and 4.2. Notice that by Lemma 3.4 and our assumption, the function $h$ defined as in the proof of Theorem 4.2 vanishes outside of a null set (a meager set), and the function $g$ constructed as in the proof of Theorem 4.1 differs from $h$ only on a null set (on a meager set, respectively).

Theorem 4.7. Let $\mathfrak{A}$ be an at most countable family of Borel measurable functions. The following are equivalent:

(i) there is a positive function $g$ such that $f+g \in \mathbf{D}$ for each $f \in \mathfrak{A}$;

(ii) there is a positive function $g \in \mathbf{B}_{2}$ such that $f+g \in \mathbf{D}$ for each $f \in \mathfrak{A}$ and $[g \neq 0]$ is a null meager set;

(iii) the inequality $(\star)$ holds for each $x \in \mathbb{R}$.

Proof. The implication (ii) $\Rightarrow$ (i) is obvious, and (i) $\Rightarrow$ (iii) follows by Theorem 4.2.

(iii) $\Rightarrow$ (ii). Let $h(x)$ equal the left-hand side of the inequality $(\star)$ if it is nonnegative, and zero otherwise. Observe that by Lemma 3.4 , the set $[h \neq 0]$ is at most countable. Thus $h \in \mathbf{B}_{2}$. Moreover for each $f \in \mathfrak{A}$ and $x \in \mathbb{R}$ we have

$$
\max \left\{\mathfrak{c}-\underline{\lim }\left(f+h, x^{-}\right), \mathfrak{c}-\underline{\lim }\left(f+h, x^{+}\right)\right\} \leq(f+h)(x) .
$$

(Cf. the proof of Theorem 4.2.) Next we proceed as in the proof of Theorem 4.1. However, we use Lemma 3.2 instead of Lemma 3.1 to find nonempty null perfect sets $Q_{\alpha n}$ (by Lemma 3.5, we may assume that each $f_{\alpha} \backslash Q_{\alpha n}$ is continuous), and we require that each function $g_{\alpha n}$ be Baire one. (See Lemma 3.7.) Then the function $g$ constructed as in the proof of Theorem 4.2 will be Baire two. 


\section{Cliquish functions}

Lemma 5.1. Let $I=[a, b]$ and $n \in \mathbb{N}$. Moreover let $f_{1}, \ldots, f_{k} \in \mathbf{C}_{q}$ be such that $\max \left\{\omega\left(f_{1}, I\right), \ldots, \omega\left(f_{k}, I\right)\right\}<1$. There exists a positive function $g \in \mathbf{B}_{1}$ such that $g=1$ on fr $I, \mathcal{C}_{g} \supset \bigcap_{i=1}^{k} \mathcal{C}_{f_{i}}$, and for each $i:\left(f_{i}+g\right)\lceil I$ is Darboux, and $\left(f_{i}+g\right)[I] \supset\left[\inf f_{i}[I]+1, \max \left\{\inf f_{i}[I]+1, n\right\}\right]$.

Proof. Put $T=\max \left\{\left|n-\inf f_{i}[I]\right|: i \in\{1, \ldots, k\}\right\}+1$. Let $\psi$ be a nonnegative continuous function such that $\psi[I]=[0, T]$ and $\psi=0$ outside of $I$. For each $i$ define $\bar{f}_{i}=\left(f_{i}+\psi\right) \cdot \chi_{I}+f_{i}(a) \cdot \chi_{(-\infty, a)}+f_{i}(b) \cdot \chi_{(b, \infty)}$. Use [12, Theorem 4] to construct a function $\bar{g} \in \mathbf{B}_{1}$ such that $\bar{f}_{i}+\bar{g} \in \mathbf{D}$ for each $i, \mathcal{C}_{\bar{g}} \supset \bigcap_{i=1}^{k} \mathrm{C}_{f_{i}}$, and $\|\bar{g}\|<1$. By its proof, we can conclude that $\bar{g}=0$ on $\{a, b\}$. Put $g=\psi+\bar{g}+1$. Observe that for each $i$, since $\bar{f}_{i}+\bar{g} \in \mathbf{D}$ and $f_{i}+g=\bar{f}_{i}+\bar{g}+1$ on $I$, so

$$
\begin{aligned}
\left(f_{i}+g\right)[I] & \supset\left(\inf \left(f_{i}+g\right)[I], \sup \left(f_{i}+g\right)[I]\right) \\
& \supset\left(f_{i}(a), \inf f_{i}[I]+\sup g[I]\right) \supset\left[\inf f_{i}[I]+1, \max \left\{\inf f_{i}[I]+1, n\right\}\right] .
\end{aligned}
$$

The other requirements are evident.

Theorem 5.2. Let $f_{1}, \ldots, f_{k} \in \mathbf{C}_{q}$. The following are equivalent:

(i) there is a positive function $g$ such that $f_{i}+g \in \mathbf{D}$ for each $i$;

(ii) there is a positive function $g$ such that $f_{i}+g \in \mathbf{D}$ for each $i$ and $\varrho_{g} \supset \bigcap_{i=1}^{k} \bigodot_{f_{i}}$;

(iii) for each $i$ and each $x \in \mathbb{R}$ we have $\max \left\{\mathfrak{c}-\underline{\lim }\left(f_{i}, x^{-}\right), \mathfrak{c}-\underline{\lim }\left(f_{i}, x^{+}\right)\right\}<\infty$.

Proof. The implication (i) $\Rightarrow$ (iii) follows by Corollary 4.3 , and (ii) $\Rightarrow$ (i) is obvious.

(iii) $\Rightarrow$ (ii). Define $A=\bigcup_{i=1}^{k}\left\{x \in \mathbb{R}: \omega\left(f_{i}, x\right) \geq 1\right\}$. Then $A$ is closed and nowhere dense. Find a family, $\left\{I_{n}: n \in \mathbb{N}\right\}$, consisting of nonoverlapping compact intervals, such that $\bigcup_{n \in \mathbb{N}} I_{n}=\mathbb{R} \backslash A$ and each $x \notin A$ belongs to $\operatorname{int}\left(I_{n} \cup I_{m}\right)$ for some $n, m \in \mathbb{N}$. For each $n \in \mathbb{N}$, since $I_{n}$ is compact and $\omega\left(f_{i}, x\right)<1$ for each $i \leq k$ and $x \in I_{n}$, we may assume that $\max \left\{\omega\left(f_{1}, I_{n}\right), \ldots, \omega\left(f_{k}, I_{n}\right)\right\}<1$.

For each $n \in \mathbb{N}$ use Lemma 5.1 to construct a positive function $g_{n} \in \mathbf{B}_{1}$ such that $g_{n}=1$ on fr $I_{n}, \mathcal{C}_{g_{n}} \supset \bigcap_{i=1}^{k} \mathcal{C}_{f_{i}}$, and for each $i:\left(f_{i}+g_{n}\right)\left\lceil I_{n}\right.$ is Darboux and $\left(f_{i}+g_{n}\right)\left[I_{n}\right] \supset\left[\inf f_{i}\left[I_{n}\right]+1, \max \left\{\inf f_{i}\left[I_{n}\right]+1, n\right\}\right]$.

Set $B=A \backslash \bigcup_{i=1}^{k} \mathcal{B}_{f_{i} \uparrow A}$. Then by Lemma 3.4, $|A \backslash B|<\mathfrak{c}$. Let $\left\{J_{p}: p \in N\right\}$ be a family consisting of all open intervals with rational end points which intersect $B$. (We have either $N=\emptyset$ or $N=\mathbb{N}$.) There is a family, $\left\{Q_{i p}: i \in\{1, \ldots, k\}, p \in N\right\}$, consisting of pairwise disjoint sets of cardinality $\mathfrak{c}$, such that each $Q_{i p}$ is a subset of $\left[f_{i}<y_{i p}\right] \cap B \cap J_{p}$, where $y_{i p}=\max \left\{\inf f_{i}\left[B \cap J_{p}\right]+p^{-1},-p\right\}$. (See Lemma 3.1.) For each $i$ and $p$ let $\psi_{i p}: Q_{i p} \rightarrow\left(y_{i p}, \infty\right)$ be a surjection. Define

$$
g(x)= \begin{cases}g_{n}(x) & \text { if } x \in I_{n}, n \in \mathbb{N}, \\ \psi_{i p}(x)-f_{i}(x) & \text { if } x \in Q_{i p}, i \in\{1, \ldots, k\}, p \in N, \\ 1 & \text { if } x \in B \backslash \bigcup_{i=1}^{k} \bigcup_{p \in N} Q_{i p},\end{cases}
$$

and $g(x)=\max \left\{\max \left\{\max \left\{\mathfrak{c}-\underline{\lim }\left(f_{i}, x^{-}\right), \mathfrak{c}-\underline{\lim }\left(f_{i}, x^{+}\right)\right\}-f_{i}(x): i \leq k\right\}, 0\right\}+1$ if $x \in A \backslash B$. It is easy to show that $g$ is the required function.

Theorem 5.3. Let $f_{1}, \ldots, f_{k} \in \mathbf{C}_{q}$ be Lebesgue measurable (Borel measurable). The following are equivalent:

(i) there is a positive function $g$ such that $f_{i}+g \in \mathbf{D}$ for each $i$; 
(ii) there is a positive Lebesgue measurable (Baire two, respectively) function $g$ such that $f_{i}+g \in \mathbf{D}$ for each $i$ and $\mathcal{C}_{g} \supset \bigcap_{i=1}^{k} \mathcal{C}_{f_{i}}$;

(iii) for each $i$ and each $x \in \mathbb{R}$ we have $\max \left\{\mathfrak{c}-\underline{\lim }\left(f_{i}, x^{-}\right), \mathfrak{c}-\underline{\lim }\left(f_{i}, x^{+}\right)\right\}<\infty$.

Proof. The implication (i) $\Rightarrow$ (iii) follows by Corollary 4.3 , and (ii) $\Rightarrow$ (i) is obvious.

(iii) $\Rightarrow$ (ii). The proof is a repetition of the argument used in Theorem 5.2. In the Lebesgue measurable case we require that each $Q_{i p}$ be a null set, so $g$ differs from a Baire one function on a null set $\bigcup_{i=1}^{k} \bigcup_{p \in N} Q_{i p} \cup(A \backslash B)$. (See Lemma 3.4.) In the Borel measurable case we use Lemma 3.2 instead of Lemma 3.1, and require that each $Q_{i p}$ be perfect. Moreover we may assume that each $f_{i} \uparrow Q_{i p}$ is continuous (Lemma 3.5) and that each $\psi_{i p}$ is Baire one (Lemma 3.7). Since by Lemma 3.4 the set $A \backslash B=\bigcup_{i=1}^{k} \mathcal{B}_{f_{i} \uparrow A}$ is at most countable, we conclude that $g \in \mathbf{B}_{2}$.

Theorem 5.4. Given a family of nonnegative functions, $\left\{g_{\alpha}: \alpha<\mathfrak{c}\right\}$, we can find a positive function $f \in \mathbf{A} \mathbf{C}_{q} \mathbf{L}$ such that $f+g_{\alpha} \notin \mathbf{D}$ for each $\alpha<\mathfrak{c}$.

Proof. Let $K$ be the set of all bilateral limit points of the Cantor ternary set. First we will define a function $\bar{f}: K \rightarrow(0,1)$ so that $\operatorname{int}\left(\bar{f}+g_{\alpha}\right)[K]=\emptyset$ for each $\alpha<\mathfrak{c}$. Arrange all elements of $K$ in a transfinite sequence, $\left\{x_{\beta}: \beta<\mathfrak{c}\right\}$. We will proceed by transfinite induction. Fix a $\beta<\mathfrak{c}$ and suppose we have already defined the function $\bar{f}$ on $\left\{x_{\gamma}: \gamma<\beta\right\}$. Let $Q_{\beta} \subset \mathbb{R} \backslash\left\{\left(\bar{f}+g_{\beta}\right)\left(x_{\gamma}\right): \gamma<\beta\right\}$ be a countable set dense in $\mathbb{R}$. Observe that $\left|B_{\beta}\right|<\mathfrak{c}$, where

$$
B_{\beta}=\bigcup_{\alpha \leq \beta}\left(\left\{y-g_{\alpha}\left(x_{\beta}\right): y \in Q_{\alpha}\right\} \cup\left\{\left(\bar{f}+g_{\alpha}\right)\left(x_{\gamma}\right)-g_{\alpha}\left(x_{\beta}\right): \gamma<\beta\right\}\right) .
$$

Choose an arbitrary $y_{\beta} \in(0,1) \backslash B_{\beta}$, and define $\bar{f}\left(x_{\beta}\right)=y_{\beta}$. Notice that for each $\alpha \leq \beta$ we have $Q_{\alpha} \cap\left(\bar{f}+g_{\alpha}\right)\left[\left\{x_{\gamma}: \gamma \leq \beta\right\}\right]=\emptyset$.

Now let $\left\{I_{m}: m \in \mathbb{N}\right\}$ be the family of all components of $\mathbb{R} \backslash K$. Define $f(x)=m$ if $x \in I_{m}$ for some $m \in \mathbb{N}$, and $f(x)=\bar{f}(x)$ otherwise. If $x \in \operatorname{cl}(K \cap(-\infty, x))$, then $0 \leq \mathfrak{c}-\underline{\lim }\left(f, x^{-}\right) \leq 1<\infty$, and if $x \in I_{m}$ for some $m \in \mathbb{N}$ and $x$ is not the left end point of $I_{m}$, then $\mathfrak{c}-\underline{\lim }\left(f, x^{-}\right)=m<\infty$. Similarly we can show that $0 \leq \mathfrak{c}-\underline{\lim }\left(f, x^{+}\right)<\infty$ for each $x \in \mathbb{R}$. By Corollary 4.5, $f \in \mathbf{A}$. It is evident that $f \in \mathbf{C}_{q} \mathbf{L}$.

Suppose $f+g_{\alpha} \in \mathbf{D}$ for some $\alpha<\mathfrak{c}$. Fix an $x \in K$. Take an open interval $I \ni x$ such that $I \cap I_{m}=\emptyset$ for $m \leq\left(f+g_{\alpha}\right)(x)+1$. Then $f+g_{\alpha} \geq f>\left(f+g_{\alpha}\right)(x)+1$ on $I \backslash K$. But $f+g_{\alpha} \in \mathbf{D}$, so $\left(f+g_{\alpha}\right)[K \cap I] \supset\left(\left(f+g_{\alpha}\right)(x),\left(f+g_{\alpha}\right)(x)+1\right)$, an impossibility.

Corollary 5.5. There is a positive function $f \in \mathbf{A C}_{q} \mathbf{L}$ such that $f+g \notin \mathbf{D}$ for each nonnegative Borel measurable function $g$.

\section{BAIRE ONE FUNCTIONS}

First let us define a classification of Baire one functions. If $\Gamma$ is a nonnegative extended real number and $A \subset \mathbb{R}$, then $\mathcal{D}_{\Gamma}(A)$ is the family of all functions $f \in \mathbf{B}_{1}$ such that

$$
\mathfrak{c}-\underline{\lim }\left(|f-f(x)| \mid A, x^{-}\right) \leq \Gamma \quad \text { whenever } x \in A \text { is a left } \mathfrak{c} \text {-limit point of } A
$$

and

$$
\mathfrak{c} \text { - } \underline{\lim }\left(|f-f(x)| \mid A, x^{+}\right) \leq \Gamma \quad \text { whenever } x \in A \text { is a right } \mathfrak{c} \text {-limit point of } A \text {; }
$$

moreover let $\mathcal{D}_{\Gamma}=\mathcal{D}_{\Gamma}(\mathbb{R})$. Then, in particular, $\mathcal{D}_{0}=\mathbf{D B}_{1}$ (see [20], [3, Theorem 6.1], or [2, Theorem 1.1, p. 9]), and $\mathcal{D}_{\infty}=\mathbf{B}_{1}$. 
Lemma 6.1. Let $\varphi \in \mathbf{D B}_{1}$ and let $P$ be a nonempty perfect set. If $\varphi$ vanishes outside of $P$, then $\varphi \in \mathcal{D}_{0}(P)$.

Proof. Let $x \in P$ be a right $c$-limit point of $P$. If $\varphi(x) \neq 0$, then find a perfect set $K$ such that $x$ is a bilateral limit point of $K$ and $\varphi\lceil K$ is continuous at $x$. (See [15], [3, Theorem 6.1], or [2, Theorem 1.1, p. 9].) Clearly we may assume that $\omega(\varphi, K)<|\varphi(x)|$. Then $K \subset P$, so $\mathfrak{c}-\underline{\lim }\left(|\varphi-\varphi(x)| \mid P, x^{+}\right)=0$.

Now let $\varphi(x)=0$ and suppose that $\mathfrak{c}$-lim $\left(|\varphi| \mid P, x^{+}\right)>0$. There is an $\varepsilon>0$ such that $|[|\varphi|<\varepsilon] \cap P \cap(x, x+\varepsilon)|<\mathfrak{c}$. Since $\varphi \in \mathbf{D}$, the set $\varphi[[x, x+\varepsilon)]$ is connected. Hence $\varphi=0$ on $[x, x+\varepsilon)$. But $x$ is a right $\mathfrak{c}$-limit point of $P$, so $\mathfrak{c}-\underline{\lim }\left(|\varphi| \uparrow P, x^{+}\right)=0$, an impossibility. Similarly we can show that $\mathfrak{c}-\underline{\lim }\left(|\varphi| \mid P, x^{-}\right)=0$ whenever $x \in P$ and $x$ is a left $\mathbf{c}$-limit point of $P$.

Corollary 6.2. Let $\varphi \in \mathbf{D B}_{1}$ and let $P$ be a nonempty perfect set. If $\varphi$ vanishes outside of $P$ and a function $f: P \rightarrow \mathbb{R}$ is continuous, then $f+\varphi, f \varphi \in \mathcal{D}_{0}(P)$.

Lemma 6.3. For each function $u$ the following are equivalent:

(i) there is a function $h \in \mathbf{B}_{1}$ such that $u \leq h$ on $\mathbb{R}$;

(ii) there is a function $f \in \mathbf{B}_{1}^{*}$ such that $u<f$ on $\mathbb{R}$;

(iii) for each nonempty closed set $P \subset \mathbb{R}$ there is an open interval $I$ such that $P \cap I \neq \emptyset$ and $u$ is bounded above on $P \cap I$.

Proof. The implication (ii) $\Rightarrow$ (i) is obvious.

(i) $\Rightarrow$ (iii). Let $x \in \mathcal{C}_{h \uparrow P}$. Then clearly $h$ is bounded on $P \cap I$ for some open interval $I \ni x$. So $u$ is bounded above on $P \cap I$.

(iii) $\Rightarrow$ (ii). Denote by $\mathcal{J}$ the family of all compact intervals, $J$, for which there is a function $f \in \mathbf{B}_{1}^{*}$ such that $u<f$ on $J$. Moreover let $G$ be the set of all $x \in \mathbb{R}$ for which there is a $\delta_{x}>0$ such that every compact interval $J \subset\left(x-\delta_{x}, x+\delta_{x}\right)$ belongs to $\mathcal{J}$.

Assertion 1. If $\left[a_{1}, a_{2}\right] \in \mathcal{J}$ and $\left[a_{2}, a_{3}\right] \in \mathcal{J}$, then $\left[a_{1}, a_{3}\right] \in \mathcal{J}$.

For $j \in\{1,2\}$ let $f_{j} \in \mathbf{B}_{1}^{*}$ witness $\left[a_{j}, a_{j+1}\right] \in \mathcal{J}$. Define $f(x)=f_{1}(x)$ if $x \leq a_{2}$, and $f(x)=f_{2}(x)$ if $x>a_{2}$. Clearly this function witnesses $\left[a_{1}, a_{3}\right] \in \mathcal{J}$.

Assertion 2. Every compact interval $J \subset G$ belongs to $\mathcal{J}$.

Indeed, the compactness of $J$ and the relation $J \subset \bigcup_{x \in J}\left(x-\delta_{x}, x+\delta_{x}\right)$ imply that there exist $x_{1}, \ldots, x_{m} \in J$ such that $J \subset \bigcup_{i=1}^{m}\left(x_{i}-\delta_{x_{i}}, x_{i}+\delta_{x_{i}}\right)$. Hence we can find nonoverlapping compact intervals $J_{1}, \ldots, J_{k} \in \mathcal{J}$ with $J=\bigcup_{j=1}^{k} J_{j}$. By Assertion 1, we obtain $J \in \mathcal{J}$.

Assertion 3. $G=\mathbb{R}$.

Indeed, suppose that the closed set $P=\mathbb{R} \backslash G$ is nonempty. There is an open interval $I$ and a $T \in \mathbb{R}$ such that $P \cap I \neq \emptyset$ and $u<T$ on $P \cap I$. Observe that $\operatorname{int}(P \cap I) \subset G$, so $P \cap I$ is nowhere dense. Take a compact interval $J \subset I$. If $J \subset G$, then by Assertion $2, J \in \mathcal{J}$. Otherwise find a family, $\left\{J_{n}: n \in \mathbb{N}\right\}$, consisting of nonoverlapping compact intervals, such that $J \backslash P=\bigcup_{n \in \mathbb{N}} J_{n}$ and each $x \in J \backslash P$ belongs to $\operatorname{int}\left(J_{n} \cup J_{m} \cup(\mathbb{R} \backslash J)\right)$ for some $n, m \in \mathbb{N}$. For each $n$ let $J_{n}=\left[a_{n}, b_{n}\right]$ and choose a function $f_{n} \in \mathbf{B}_{1}^{*}$ witnessing $J_{n} \in \mathcal{J}$. Define $f(x)=f_{n}(x)$ if $x \in\left[a_{n}, b_{n}\right)$ for some $n \in \mathbb{N}$, and $f(x)=T$ otherwise. Clearly this function witnesses $J \in \mathcal{J}$. Hence $I \subset G$ and $P \cap I \subset P \cap G=\emptyset$, an impossibility.

Finally, for each $z \in \mathbb{Z}$ use Assertions 3 and 2 to construct a function $f_{z} \in \mathbf{B}_{1}^{*}$ witnessing $[z, z+1] \in \mathcal{J}$. Define $f(x)=f_{z}(x)$ if $x \in[z, z+1)$ for some $z \in \mathbb{Z}$. Then clearly $f \in \mathbf{B}_{1}^{*}$ and $u<f$ on $\mathbb{R}$. 
Lemma 6.4. Let $g, f_{1}, \ldots, f_{k} \in \mathbf{B}_{1}$. Moreover assume that $g>0$ on a compact interval $I$, and let $d_{i} \geq c_{i}>\mathfrak{c}-\inf \left(f_{i}, I\right)$ for $i \in\{1, \ldots, k\}$. Then there exists a function $\tilde{g} \in \mathbf{B}_{1}$ such that $\tilde{g}>0$ on $I, \tilde{g}=g$ outside of int $I$, and for each $i \in\{1, \ldots, k\}: \mathfrak{c}-\underline{\lim }\left(\left|f_{i}+\tilde{g}-f_{i}(x)-\tilde{g}(x)\right|, x^{-}\right)=\mathfrak{c}-\underline{\lim }\left(\left|f_{i}+g-f_{i}(x)-g(x)\right|, x^{-}\right)$ and $\mathfrak{c}-\underline{\lim }\left(\left|f_{i}+\tilde{g}-f_{i}(x)-\tilde{g}(x)\right|, x^{+}\right)=\mathfrak{c}-\underline{\lim }\left(\left|f_{i}+g-f_{i}(x)-g(x)\right|, x^{+}\right)$for each $x \in \mathbb{R}$, and $\left(f_{i}+\tilde{g}\right)[I] \supset\left[c_{i}, d_{i}\right]$.

Proof. I. First choose pairwise disjoint nonempty perfect sets $P_{1}, \ldots, P_{k}$ such that $P_{i} \subset\left[f_{i}<c_{i}\right] \cap \operatorname{int} I$ for each $i$. By Lemma 3.5, we may assume that $g\left\lceil P_{i}\right.$ and $f_{j}\left\lceil P_{i}\right.$ are continuous for each $i$ and $j$. Use Lemma 3.6 to construct a Darboux Baire one function $\hat{g}: \mathbb{R} \rightarrow(0,1]$ such that $\hat{g}=1$ outside of $\bigcup_{i=1}^{k} P_{i}$ and inf $\hat{g}\left[P_{i}\right]=0$ for each $i$. Define $\bar{g}=g \hat{g}$.

It is obvious that $\bar{g} \in \mathbf{B}_{1}, \bar{g}>0$ on $I$, and $\bar{g}=g$ outside of int $I$. Fix an $i \in\{1, \ldots, k\}$. The function $g \mid P_{i}$ is continuous, so it is bounded above. Let $T_{i}=\sup g\left[P_{i}\right]$ and $\varepsilon_{i}=c_{i}-\sup f_{i}\left[P_{i}\right]>0$. Recall that $\inf \hat{g}\left[P_{i}\right]=0$ and $\hat{g} \in \mathbf{D}$. Thus $\left|\left[\hat{g}<\varepsilon_{i} / T_{i}\right] \cap P_{i}\right|=\mathfrak{c}$. Hence $\left|\left[f_{i}+\bar{g}<c_{i}\right] \cap P_{i}\right|=\mathfrak{c}$ and $\mathfrak{c}$-inf $\left(f_{i}+\bar{g}, I\right)<c_{i}$.

Let $x \in \mathbb{R}$. If $x$ is not a left $\mathfrak{c}$-limit point of $\bigcup_{j=1}^{k} P_{j}$, then $\bar{g}=g$ on $(x-\delta, x]$ for some $\delta>0$, so $\mathfrak{c}-\underline{\lim }\left(\left|f_{i}+\bar{g}-f_{i}(x)-\bar{g}(x)\right|, x^{-}\right)=\mathfrak{c}-\underline{\lim }\left(\left|f_{i}+g-f_{i}(x)-g(x)\right|, x^{-}\right)$. Otherwise by Corollary 6.2 , we obtain

$$
\mathfrak{c}-\underline{\lim }\left(\left|f_{i}+\bar{g}-f_{i}(x)-\bar{g}(x)\right|, x^{-}\right)=0=\mathfrak{c}-\underline{\lim }\left(\left|f_{i}+g-f_{i}(x)-g(x)\right|, x^{-}\right) .
$$

Similarly $\mathfrak{c}-\underline{\lim }\left(\left|f_{i}+\bar{g}-f_{i}(x)-\bar{g}(x)\right|, x^{+}\right)=\mathfrak{c}-\underline{\lim }\left(\left|f_{i}+g-f_{i}(x)-g(x)\right|, x^{+}\right)$.

II. Now choose pairwise disjoint nonempty perfect sets $Q_{1}, \ldots, Q_{k}$ such that $Q_{i} \subset\left[f_{i}+\bar{g}<c_{i}\right] \cap \operatorname{int} I$ for each $i$. By Lemma 3.5, we may assume that $\left(f_{i}+\bar{g}\right)\left\lceil Q_{i}\right.$ is continuous for each $i$. For each $i$ let $\psi_{i}$ be a continuous function mapping $Q_{i}$ onto $\left[c_{i}, d_{i}\right]$. Use the Tietze extension theorem to construct a nonnegative continuous function $\psi$ such that $\psi=\psi_{i}-f_{i}-\bar{g}$ on $Q_{i}$ for each $i$, and $\psi=0$ outside of $I$.

III. Finally, define $\tilde{g}=\bar{g}+\psi$. Clearly this function has all required properties.

Proposition 6.5. Let $h, f_{1}, \ldots, f_{k} \in \mathbf{B}_{1}$. Moreover, assume that for each $i$ and each $x \in \mathbb{R}$ we have $\max \left\{\mathfrak{c}-\underline{\underline{\lim }}\left(f_{i}, x^{-}\right)\right.$, $\left.\mathfrak{c} \underline{\underline{\lim }}\left(f_{i}, x^{+}\right)\right\} \leq h(x)$. Then there is a positive function $g \in \mathbf{B}_{1}$ such that $f_{i}+g \in \overline{\mathcal{D}_{1}}$ for each $i$.

Proof. Let $f \in \mathbf{B}_{1}^{*}$ be such that $f>\left|f_{i}\right|+|h|$ on $\mathbb{R}$ for each $i$. (See Lemma 6.3.) Put $C=\bigcap_{i=1}^{k} \mathcal{e}_{f_{i}}$. Denote by $\mathcal{J}$ the family of all compact intervals, $J$, such that fr $J \subset C$ and there is a positive function $g \in \mathbf{B}_{1}$ with $g=1$ on fr $J$ and $f_{i}+g \in \mathcal{D}_{1}(J)$ for each $i$. Moreover let $G$ be the set of all $x \in \mathbb{R}$ for which there is a $\delta_{x}>0$ such that every compact interval $J \subset\left(x-\delta_{x}, x+\delta_{x}\right)$ with fr $J \subset C$ belongs to $\mathcal{J}$. Observe that by Lemma 5.1 we have $C \subset G$, so $G$ is dense in $\mathbb{R}$.

The first two assertions can be proved in essentially the same way as in the proof of Lemma 6.3.

Assertion 1. If $\left[a_{1}, a_{2}\right] \in \mathcal{J}$ and $\left[a_{2}, a_{3}\right] \in \mathcal{J}$, then $\left[a_{1}, a_{3}\right] \in \mathcal{J}$.

Assertion 2. Every compact interval $J \subset G$ belongs to $\mathcal{J}$.

Assertion 3. $G=\mathbb{R}$.

Indeed, suppose that the closed set $P=\mathbb{R} \backslash G$ is nonempty. Let $x_{0} \in P$ and $\delta>0$ be such that $f \uparrow\left(P \cap\left(x_{0}-\delta, x_{0}+\delta\right)\right)$ is continuous and $\omega\left(f_{i}, P \cap\left(x_{0}-\delta, x_{0}+\delta\right)\right)<1$ for each $i$. Take a compact interval $J=[a, b] \subset\left(x_{0}-\delta, x_{0}+\delta\right)$ with $a, b \in C$. If $J \subset G$, then by Assertion 2, we have $J \in \mathcal{J}$. Otherwise write $J \backslash P$ as the union of a family, $\left\{J_{n}: n \in \mathbb{N}\right\}$, consisting of nonoverlapping compact intervals, such that 
each $x \in J \backslash P$ belongs to $\operatorname{int}\left(J_{n} \cup J_{m} \cup(\mathbb{R} \backslash J)\right)$ for some $n, m \in \mathbb{N}$. For each $n \in \mathbb{N}$ use Assertion 2 to find a positive function $g_{n} \in \mathbf{B}_{1}$ witnessing $J_{n} \in \mathcal{J}$. We may assume that $\left(f_{i}+g_{n}\right)\left[J_{n}\right] \supset\left[c_{i n}, d_{i n}\right]$, where $c_{i n}=\max \left\{\mathfrak{c}\right.$-inf $\left.\left(f_{i}, J_{n}\right)+n^{-1},-n\right\}$ and $d_{i n}=\max \left\{c_{i n}, n\right\}$. (See Lemma 6.4.)

Define $g(x)=g_{n}(x)$ if $x \in J_{n}$ for some $n \in \mathbb{N}$, and $g(x)=f(x)$ otherwise. Clearly $g$ is well defined and Baire one, $g>0$ on $\mathbb{R}$, and $g=1$ on fr $J$. (Recall that $a, b \in C \subset \mathbb{R} \backslash P$.) Fix an $i \leq k$ and let $x \in(a, b]$. If $x \notin P$, then there is an $n \in \mathbb{N}$ such that $x \in J_{n}$ and $x$ is not the left end point of $J_{n}$. Since $f_{i}+g_{n} \in \mathcal{D}_{1}\left(J_{n}\right)$, $\mathfrak{c}-\underline{\lim }\left(\left|f_{i}+g-f_{i}(x)-g(x)\right|, x^{-}\right) \leq 1$. If $x \in P$ and $x$ is not a left $\mathfrak{c}$-limit point of $P$, then by Lemma 3.3, for each $\delta>0$ we have $\left(f_{i}+g\right)[(x-\delta, x)] \supset\left(\mathfrak{c}-\underline{\underline{\lim }}\left(f_{i}, x^{-}\right), \infty\right)$. As $\left(f_{i}+g\right)(x)>h(x) \geq \mathfrak{c}-\underline{\lim }\left(f_{i}, x^{-}\right)$, we get $\mathfrak{c}-\underline{\lim }\left(\left|f_{i}+g-f_{i}(x)-g(x)\right|, x^{-}\right)=0$. Finally if $x \in P$ and $x$ is a left c-limit point of $P$, then using the fact that $f \uparrow(P \cap J)$ is continuous, we get $\mathfrak{c}-\underline{\lim }\left(\left|f_{i}+g-f_{i}(x)-g(x)\right|, x^{-}\right) \leq \omega\left(f_{i}, P \cap J\right)<1$.

Proceeding similarly, we can prove that $\mathfrak{c}-\underline{\lim }\left(\left|f_{i}+g-f_{i}(x)-g(x)\right|, x^{+}\right) \leq 1$ for each $x \in[a, b)$. So $f_{i}+g \in \mathcal{D}_{1}(J)$. It follows that $x_{0} \in P \cap G=\emptyset$, an impossibility. This contradiction proves that $G=\mathbb{R}$.

For each $z \in \mathbb{Z}$ construct a function $g_{z} \in \mathbf{B}_{1}$ witnessing $[z, z+1] \in \mathcal{J}$. (We use Assertions 3 and 2.) Define $g(x)=g_{z}(x)$ if $x \in[z, z+1]$ for some $z \in \mathbb{Z}$. One can easily verify that $g$ has all required properties.

Lemma 6.6. Let $J$ be an interval and let $f \in \mathcal{D}_{\tau}(J)$ for some $\tau>0$. For each $y \in(\mathfrak{c}-\inf (f, J)-\tau, \mathfrak{c}-\sup (f, J)+\tau)$ and each $\varepsilon>0$ there is a $z \in(y-\tau, y+\tau)$ such that $|[|f-z|<\varepsilon] \cap J|=\mathfrak{c}$.

Proof. If $y \leq \mathfrak{c}$-inf $(f, J)$, then $\mathfrak{c}$-inf $(f, J)>-\infty$ and we can define $z=\mathfrak{c}$-inf $(f, J)$. We proceed similarly if $y \geq \mathfrak{c}$-sup $(f, J)$. So assume $y \in(\mathfrak{c}-\inf (f, J), \mathfrak{c}-\sup (f, J))$. Suppose that $|[|f-z|<\varepsilon] \cap J|<\mathfrak{c}$ for each $z \in(y-\tau, y+\tau)$. Observe that the interval $(y-\tau-\varepsilon, y+\tau+\varepsilon)$ can be written as the countable union of intervals of length $2 \varepsilon$, each of which is centered at an internal point of $(y-\tau, y+\tau)$. Thus $|[|f-y|<\tau+\varepsilon] \cap J|<\mathfrak{c}$. But $f \in \mathcal{D}_{\tau}(J)$, so $[|f-y|<\varepsilon] \cap J=\emptyset$.

Define $\bar{f}=\max \{\min \{f, y+\varepsilon\}, y-\varepsilon\}$. Using the fact that $f \in \mathcal{D}_{\tau}(J)$, one can easily show that $\bar{f} \in \mathcal{D}_{0}(J)$, i.e., that $\bar{f}\lceil J$ is Darboux. It follows that either $f \leq y-\varepsilon$ on $J$ and $y>\mathfrak{c}-\sup (f, J)$, or $f \geq y+\varepsilon$ on $J$ and $y<\mathfrak{c}-\inf (f, J)$. Both cases are impossible. This completes the proof.

Lemma 6.7. Let $f \in \mathbf{B}_{1}, P$ be a nonempty perfect set, $x \in P$, and $|f-f(x)|<\varepsilon$ on $P$. For each $\tilde{\tau}>0$ there is a function $\psi \in \mathbf{D B}_{1}$ such that $\psi$ vanishes outside of $P,(f+\psi)[P] \supset[f(x)-\tilde{\tau}, f(x)+\tilde{\tau}]$, and $|\psi|<\tilde{\tau}+\varepsilon$ on $P$.

Proof. Without loss we may assume that $P$ is nowhere dense and $f \nmid P$ is continuous. (See Lemma 3.5.) Let $\widetilde{P}$ be a nowhere dense perfect set of positive measure and let $u: \mathbb{R} \rightarrow \mathbb{R}$ be a homeomorphism with $u[P]=\widetilde{P}$. Let $\widetilde{Q}$ be a nonempty perfect set consisting of points of density of $\widetilde{P}$. Use [21, Lemma 12] to find an approximately continuous function $\tilde{\varphi}: \mathbb{R} \rightarrow[0,1]$ such that $\tilde{\varphi}=1$ on $\widetilde{Q}$ and $\tilde{\varphi}=0$ outside of $\widetilde{P}$. Let $\tilde{f}$ be a continuous extension of $f \circ u^{-1} \uparrow \widetilde{P}$ to the whole real line. Find a continuous function $\tilde{\psi}: \mathbb{R} \rightarrow[f(x)-\tilde{\tau}, f(x)+\tilde{\tau}]$ with $\tilde{\psi}[\widetilde{Q}]=[f(x)-\tilde{\tau}, f(x)+\tilde{\tau}]$. Define $\psi=((\tilde{\psi}-\tilde{f}) \cdot \tilde{\varphi}) \circ u$. Then

$$
(f+\psi)[P] \supset(f+\psi)\left[u^{-1}(\widetilde{Q})\right]=\tilde{\psi}[\widetilde{Q}]=[f(x)-\tilde{\tau}, f(x)+\tilde{\tau}],
$$


and since the function $\psi \circ u^{-1}$ is approximately continuous, $\psi \in \mathbf{D B}_{1}$. (See [14] or [17, Theorem 2].) Finally if $t \notin P$, then $\tilde{\varphi}(u(t))=0$ and $\psi(t)=0$, and $t \in P$ implies $|\psi(t)| \leq \tilde{\tau}+|f(t)-f(x)|<\tilde{\tau}+\varepsilon$. This completes the proof.

Proposition 6.8. Let $\tau>\varepsilon>0$ and $f_{1}, \ldots, f_{k} \in \mathcal{D}_{\tau}$. Then there is a positive function $g \in \mathbf{B}_{1}$ such that $f_{i}+g \in \mathcal{D}_{\varepsilon}$ for each $i$ and $g<2 \tau+2 \varepsilon$ on $\mathbb{R}$.

Proof. Denote by $\mathcal{J}$ the family of all compact intervals, $J$, with the property that for each $\eta \in(\tau, \tau+\varepsilon)$ and any real numbers $\mathfrak{c}-\sup \left(f_{i}, J\right) \geq d_{i} \geq c_{i} \geq \mathfrak{c}-\inf \left(f_{i}, J\right)$ $(i \in\{1, \ldots, k\})$ there is a function $g \in \mathbf{B}_{1}$ such that $|g|<\tau+\varepsilon$ on $J, g=0$ outside of int $J$, and for each $i: f_{i}+g \in \mathcal{D}_{\varepsilon}(J)$ and $\left(f_{i}+g\right)[J] \supset\left(c_{i}-\eta, d_{i}+\eta\right)$. Moreover let $G$ be the set of all $x \in \mathbb{R}$ for which there is a $\delta_{x}>0$ such that every compact interval $J \subset\left(x-\delta_{x}, x+\delta_{x}\right)$ belongs to $\mathcal{J}$.

The first two assertions can be proved essentially in the same way as in the proof of Lemma 6.3.

Assertion 1. If $\left[a_{1}, a_{2}\right] \in \mathcal{J}$ and $\left[a_{2}, a_{3}\right] \in \mathcal{J}$, then $\left[a_{1}, a_{3}\right] \in \mathcal{J}$.

Fix $\eta \in(\tau, \tau+\varepsilon)$ and real numbers $\mathfrak{c}$-sup $\left(f_{i},\left[a_{1}, a_{3}\right]\right) \geq d_{i} \geq c_{i} \geq \mathfrak{c}$-inf $\left(f,\left[a_{1}, a_{3}\right]\right)$ $(i \in\{1, \ldots, k\})$. For $j \in\{1,2\}$ let $g_{j} \in \mathbf{B}_{1}$ be such that $\left|g_{j}\right|<\tau+\varepsilon$ on $\left[a_{j}, a_{j+1}\right]$, $g_{j}=0$ outside of $\left(a_{j}, a_{j+1}\right)$, and for each $i \leq k: f_{i}+g_{j} \in \mathcal{D}_{\varepsilon}\left(\left[a_{j}, a_{j+1}\right]\right)$ and $\left(f_{i}+g_{j}\right)\left[\left[a_{j}, a_{j+1}\right]\right] \supset\left(c_{i j}-\eta, d_{i j}+\eta\right)$, where

$$
\begin{aligned}
c_{i j} & = \begin{cases}\mathfrak{c}-\inf \left(f_{i},\left[a_{j}, a_{j+1}\right]\right) & \text { if } \mathfrak{c}-\inf \left(f_{i},\left[a_{j}, a_{j+1}\right]\right)>-\infty, \\
\min \left\{c_{i}, f_{i}\left(a_{2}\right)\right\} & \text { otherwise, }\end{cases} \\
d_{i j} & = \begin{cases}\mathfrak{c}-\sup \left(f_{i},\left[a_{j}, a_{j+1}\right]\right) & \text { if } \mathfrak{c}-\sup \left(f_{i},\left[a_{j}, a_{j+1}\right]\right)<\infty, \\
\max \left\{d_{i}, f_{i}\left(a_{2}\right)\right\} & \text { otherwise. }\end{cases}
\end{aligned}
$$

Define $g(x)=g_{1}(x)$ if $x \leq a_{2}$, and $g(x)=g_{2}(x)$ if $x>a_{2}$. Then clearly $g \in \mathbf{B}_{1}$, $|g|<\tau+\varepsilon$ on $\left[a_{1}, a_{3}\right], g=0$ outside of $\left(a_{1}, a_{3}\right)$, and $f_{i}+g \in \mathcal{D}_{\varepsilon}\left(\left[a_{1}, a_{3}\right]\right)$ for each $i$. Fix an $i \leq k$. Since $f_{i} \in \mathcal{D}_{\tau}$,

$$
\max \left\{c_{i 1}-\eta, c_{i 2}-\eta\right\}<f_{i}\left(a_{2}\right)<\min \left\{d_{i 1}+\eta, d_{i 2}+\eta\right\} .
$$

Hence by Lemma 3.3, we obtain

$$
\left(f_{i}+g\right)\left[\left[a_{1}, a_{3}\right]\right] \supset\left(\min \left\{c_{i 1}, c_{i 2}\right\}-\eta, \max \left\{d_{i 1}, d_{i 2}\right\}+\eta\right) \supset\left(c_{i}-\eta, d_{i}+\eta\right) .
$$

Thus $\left[a_{1}, a_{3}\right] \in \mathcal{J}$.

Assertion 2. Every compact interval $J \subset G$ belongs to $\mathcal{J}$.

Assertion 3. $G=\mathbb{R}$.

By way of contradiction, suppose that the closed set $P=\mathbb{R} \backslash G$ is nonempty. Let $x_{0} \in \bigcap_{i=1}^{k} \mathcal{C}_{f_{i} \uparrow P}$. Let $\delta>0$ be such that $\omega\left(f_{i}, P \cap\left(x_{0}-\delta, x_{0}+\delta\right)\right)<\varepsilon$ for $i \leq k$. Take a compact interval $J=[a, b] \subset\left(x_{0}-\delta, x_{0}+\delta\right)$. If $J \subset G$, then by Assertion 2, we have $J \in \mathcal{J}$. Otherwise fix an $\eta \in(\tau, \tau+\varepsilon)$ and real numbers $\mathfrak{c}-\sup \left(f_{i}, J\right) \geq d_{i} \geq c_{i} \geq \mathfrak{c}-\inf \left(f_{i}, J\right)(i \in\{1, \ldots, k\})$. Set $\tilde{\eta}=(\tau+\varepsilon-\eta) / 4$.

Fix an $i \leq k$. Since the interval $\left[c_{i}-\tau+\tilde{\eta}, d_{i}+\tau-\tilde{\eta}\right]$ is compact, there are $z_{i 1}, \ldots, z_{i p_{i}} \in \mathbb{R}$ such that $\left[c_{i}-\tau+\tilde{\eta}, d_{i}+\tau-\tilde{\eta}\right] \subset \bigcup_{s=1}^{p_{i}}\left(z_{i s}-\tau, z_{i s}+\tau\right)$ and $\left|\left[\left|f_{i}-z_{i s}\right|<\tilde{\eta}\right] \cap J\right|=\mathfrak{c}$ for each $s$. (We use Lemma 6.6.) Notice that $\left[c_{i}-\eta, d_{i}+\eta\right] \subset \bigcup_{s=1}^{p_{i}}\left(z_{i s}-\eta-\tilde{\eta}, z_{i s}+\eta+\tilde{\eta}\right)$.

Write $J \backslash P$ as the union of a family, $\left\{J_{n}: n \in N\right\}$, consisting of nonoverlapping compact intervals, such that each $x \in J \backslash P$ belongs to $\operatorname{int}\left(J_{n} \cup J_{m} \cup(\mathbb{R} \backslash J)\right)$ for some $n, m \in N$. (We have either $N=\emptyset$ or $N=\mathbb{N}$.) By Lemma 3.2, there is a family, $\left\{Q_{i s}: i \in\{1, \ldots, k\}, s \in\left\{1, \ldots, p_{i}\right\}\right\}$, consisting of pairwise disjoint 
nonempty perfect sets, such that $Q_{i s} \subset\left[\left|f_{i}-z_{i s}\right|<\tilde{\eta}\right] \cap J$ for each $i$ and $s$. We may assume that for each $i$ and $s$, either $Q_{i s} \subset J_{n}$ for some $n \in N$ or $Q_{i s} \subset P \backslash \mathcal{B} \chi_{P \cap J}$ (see Lemma 3.3), and $f_{j} \uparrow Q_{i s}$ is continuous for each $j$ (see Lemma 3.5). For each $i$ and $s$, if $Q_{i s} \subset P$, then find a function $\psi_{i s} \in \mathbf{D B}_{1}$ such that $\psi_{i s}$ vanishes outside of $Q_{i s},\left(f_{i}+\psi_{i s}\right)\left[Q_{i s}\right] \supset\left[z_{i s}-\eta-\tilde{\eta}, z_{i s}+\eta+\tilde{\eta}\right]$, and $\left|\psi_{i s}\right|<\tau+\varepsilon$ on $Q_{i s}$. (Use Lemma 6.7 with $\tilde{\tau}=\eta+2 \tilde{\eta}$.) If $Q_{i s} \cap P=\emptyset$, then let $\psi_{i s}=0$ on $\mathbb{R}$.

For each $n \in N$ construct a function $g_{n} \in \mathbf{B}_{1}$ such that $\left|g_{n}\right|<\tau+\varepsilon$ on $J_{n}$, $g_{n}=0$ outside of int $J_{n}$, and for each $i \in\{1, \ldots, k\}: f_{i}+g_{n} \in \mathcal{D}_{\varepsilon}\left(J_{n}\right)$ and $\left(f_{i}+g_{n}\right)\left[J_{n}\right] \supset\left(c_{i n}-\eta-2 \tilde{\eta}, d_{i n}+\eta+2 \tilde{\eta}\right)$, where

$$
\begin{aligned}
c_{i n} & = \begin{cases}\mathfrak{c}-\inf \left(f, J_{n}\right) & \text { if } \mathfrak{c} \text {-inf }\left(f, J_{n}\right)>-\infty, \\
-\sum_{s=1}^{p_{i}}\left|z_{i s}\right|-\max |f|\left[\operatorname{fr} J_{n}\right]-n & \text { otherwise, }\end{cases} \\
d_{\text {in }} & =\left\{\begin{array}{cl}
\mathfrak{c}-\sup \left(f, J_{n}\right) & \text { if } \mathfrak{c}-\sup \left(f, J_{n}\right)<\infty, \\
\sum_{s=1}^{p_{i}}\left|z_{i s}\right|+\max |f|\left[\operatorname{fr} J_{n}\right]+n & \text { otherwise }
\end{array}\right.
\end{aligned}
$$

Put $\hat{g}(x)=g_{n}(x)$ if $x \in J_{n}$ for some $n \in N$, and $\hat{g}(x)=0$ otherwise. Define $g=\sum_{i=1}^{k} \sum_{s=1}^{p_{i}} \psi_{i s}+\hat{g}$. Clearly $g$ is well defined and Baire one, $|g|<\tau+\varepsilon$ on $J$, and $g=0$ outside of int $J$. Fix an $i \leq k$.

Let $x \in(a, b]$. If $x \notin P$, then there is an $n \in N$ such that $x \in J_{n}$ and $x$ is not the left end point of $J_{n}$. Using the fact that $f_{i}+g_{n} \in \mathcal{D}_{\varepsilon}\left(J_{n}\right)$, we obtain $\mathfrak{c}-\underline{\lim }\left(\left|f_{i}+g-f_{i}(x)-g(x)\right|, x^{-}\right) \leq \varepsilon$. If $x \in P$ and $x$ is not a left $\mathfrak{c}$-limit point of $P$, then $x \in \mathcal{B} \chi_{P \cap J}$, and by Lemma 3.3, for each $\delta>0$ we have

$$
\left(f_{i}+g\right)[(x-\delta, x)] \supset\left(\mathfrak{c}-\underline{\lim }\left(f_{i}, x^{-}\right)-\eta-2 \tilde{\eta}, \mathfrak{c}-\overline{l i m}\left(f_{i}, x^{-}\right)+\eta+2 \tilde{\eta}\right) .
$$

(Cf. also the proof of Assertion 1.) Using the facts that $g(x)=0$ and $f_{i} \in \mathcal{D}_{\tau}$, we get $\mathfrak{c}-\underline{\underline{i m}}\left(\left|f_{i}+g-f_{i}(x)-g(x)\right|, x^{-}\right)=0$.

If $x \in P$ and $x$ is a left $\mathfrak{c}$-limit point of $P \cap \bigcup_{j=1}^{k} \bigcup_{s=1}^{p_{j}} Q_{j s}$, then by Corollary 6.2, we obtain $\mathfrak{c}-\underline{\lim }\left(\left|f_{i}+g-f_{i}(x)-g(x)\right|, x^{-}\right)=0$.

Finally if $x \in P, x$ is a left $\mathfrak{c}$-limit point of $P$, and $x$ is not a left $\mathfrak{c}$-limit point of $P \cap \bigcup_{j=1}^{k} \bigcup_{s=1}^{p_{j}} Q_{j s}$, then $g=0$ on $P \cap(x-\delta, x]$ for some $\delta>0$. Using the fact that $\omega\left(f_{i}, P \cap J\right)<\varepsilon$, we obtain $\mathfrak{c}-\underline{\lim }\left(\left|f_{i}+g-f_{i}(x)-g(x)\right|, x^{-}\right)<\varepsilon$.

Proceeding similarly, we can prove that $\mathfrak{c}-\underline{\lim }\left(\left|f_{i}+g-f_{i}(x)-g(x)\right|, x^{+}\right) \leq \varepsilon$ for each $x \in[a, b)$. Hence $f_{i}+g \in \mathcal{D}_{\varepsilon}(J)$.

Since $Q_{i s} \subset\left[\left|f_{i}-z_{i s}\right|<\tilde{\eta}\right]$, it follows that $z_{i s} \in\left(c_{i n}-\tilde{\eta}, d_{i n}+\tilde{\eta}\right)$ whenever $Q_{i s} \subset J_{n}$. Thus $\left(f_{i}+g\right)[J] \supset \bigcup_{s=1}^{p_{i}}\left(z_{i s}-\eta-\tilde{\eta}, z_{i s}+\eta+\tilde{\eta}\right) \supset\left(c_{i}-\eta, d_{i}+\eta\right)$.

We have shown that $J \in \mathcal{J}$. It follows that $x_{0} \in P \cap G=\emptyset$. This contradiction proves $G=\mathbb{R}$.

For each $z \in \mathbb{Z}$ use Assertion 3 and construct a function $g_{z} \in \mathbf{B}_{1}$ such that $\left|g_{z}\right|<\tau+\varepsilon$ on $[z, z+1], g_{z}=0$ on $\{z, z+1\}$, and $f_{i}+g_{z} \in \mathcal{D}_{\varepsilon}([z, z+1])$ for each $i$. Define $g(x)=g_{z}(x)+\tau+\varepsilon$ if $x \in[z, z+1]$ for some $z \in \mathbb{Z}$. This function obviously has all required properties.

Theorem 6.9. Let $f_{1}, \ldots, f_{k} \in \mathbf{B}_{1}$. The following are equivalent:

(i) there exists a positive function $g \in \mathbf{B}_{1}$ such that $f_{i}+g \in \mathbf{D}$ for each $i$;

(ii) there exists a function $h \in \mathbf{B}_{1}$ such that for each $i$ and each $x \in \mathbb{R}$ we have $\max \left\{\mathfrak{c}-\underline{\lim }\left(f_{i}, x^{-}\right), \mathfrak{c}-\underline{\lim }\left(f_{i}, x^{+}\right)\right\} \leq h(x)$.

Proof. (i) $\Rightarrow$ (ii). Let $h=\max \left\{f_{1}, \ldots, f_{k}\right\}+g$. Fix an $i \leq k$ and an $x \in \mathbb{R}$. Then

$$
\mathfrak{c}-\underline{\lim }\left(f_{i}, x^{-}\right) \leq \mathfrak{c}-\underline{\lim }\left(f_{i}+g, x^{-}\right) \leq\left(f_{i}+g\right)(x) \leq h(x) .
$$


Similarly we can show that $\mathfrak{c}-\underline{\lim }\left(f_{i}, x^{+}\right) \leq h(x)$.

(ii) $\Rightarrow$ (i). By Proposition 6.5 , there is a positive function $g_{1} \in \mathbf{B}_{1}$ such that $f_{i}+g_{1} \in \mathcal{D}_{1}$ for each $i$. For $n \geq 2$ apply Proposition 6.8 to construct a positive function $g_{n} \in \mathbf{B}_{1}$ such that $g_{n}-g_{n-1}<2 \cdot 2^{2-n}+2 \cdot 2^{1-n}$ on $\mathbb{R}$ and $f_{i}+g_{n} \in \mathcal{D}_{2^{1-n}}$ for each $i$. Define $g=\lim _{n \rightarrow \infty} g_{n}$. This sequence is uniformly convergent, so $g \in \mathbf{B}_{1}$. Clearly $g>0$ on $\mathbb{R}$. Fix an $i \leq k$. For each $x \in \mathbb{R}$ and $n \in \mathbb{N}$ we have

$$
\begin{aligned}
\mathfrak{c}-\underline{\lim }(\mid f+g-f & \left.(x)-g(x) \mid, x^{-}\right) \\
& \leq \mathfrak{c}-\underline{\lim }\left(\left|f+g_{n}-f(x)-g_{n}(x)\right|, x^{-}\right)+2\left\|g-g_{n}\right\| \\
& \leq 2^{1-n}+2 \sum_{m>n}\left\|g_{m}-g_{m-1}\right\| \\
& \leq 2^{1-n}+2 \sum_{m>n}\left(2 \cdot 2^{2-m}+2 \cdot 2^{1-m}\right)=26 \cdot 2^{-n}
\end{aligned}
$$

similarly we can show that $\mathfrak{c}-\underline{\lim }\left(|f+g-f(x)-g(x)|, x^{+}\right) \leq 26 \cdot 2^{-n}$. Hence $f_{i}+g \in \bigcap_{n \in \mathbb{N}} \mathcal{D}_{26 \cdot 2^{-n}}=\mathcal{D}_{0}=\mathbf{D B}_{1}$. This completes the proof.

Proposition 6.10. There is a positive function $f \in \mathbf{A B}_{1}^{*}$ such that for each function $\psi \in \mathbf{B}_{1}$ the condition " $f<\psi$ on $\mathbb{R}$ " implies " $\psi \notin \mathbf{D}$ ".

Proof. Let $F$ be the Cantor ternary set, and let $\mathcal{J}=\left\{\left(a_{n}, b_{n}\right): n \in \mathbb{N}\right\}$ and $\mathcal{J}$ be disjoint families of components of $\mathbb{R} \backslash F$ such that $F=(\mathrm{cl} \cup \mathcal{J}) \cap(\operatorname{cl} \bigcup \mathcal{J})$. Define $f(x)=n$ if $x \in\left(a_{n}, b_{n}\right)$ for some $n \in \mathbb{N}$, and $f(x)=1$ otherwise. If $x \in\left(a_{n}, b_{n}\right]$ for some $n \in \mathbb{N}$, then $\mathfrak{c}-\underline{\lim }\left(f, x^{-}\right)=n$, and if $x \notin \bigcup_{n \in \mathbb{N}}\left(a_{n}, b_{n}\right]$, then $\mathfrak{c}-\underline{\lim }\left(f, x^{-}\right)=1$. Similarly we can show that $1 \leq \mathfrak{c}-\underline{\lim }\left(f, x^{+}\right)<\infty$ for each $x \in \mathbb{R}$. By Corollary 4.5, we obtain $f \in \mathbf{A}$. It is evident that $f \in \mathbf{B}_{1}^{*}$.

On the other hand, the function $u(x)=\max \left\{\mathfrak{c}-\underline{\lim }\left(f_{i}, x^{-}\right), \mathfrak{c}-\underline{\lim }\left(f_{i}, x^{+}\right)\right\}$is unbounded above on each portion of $F$. So by Lemma 6.3 and Theorem 6.9, we obtain that $f+g \notin \mathbf{D}$ for each positive function $g \in \mathbf{B}_{1}$.

\section{Open PROBlems}

Problem 7.1. Is it consistent with $Z F C$ that there is an uncountable cardinal $\kappa$ such that the assertion of Theorem 4.7 holds for each family of Borel measurable functions $\mathfrak{A}$ with $|\mathfrak{A}| \leq \kappa$ ?

We say that a function $f$ is almost continuous in the sense of Stallings [19] if every open set $G \subset \mathbb{R}^{2}$ containing the graph of $f$ contains the graph of some continuous function. Recall that almost continuous functions are Darboux, but the converse is not true. However, in Baire class one these two notions coincide [1].

Problem 7.2. Characterize averages of comparable almost continuous functions.

\section{REFERENCES}

1. J. B. Brown, Almost continuous Darboux functions and Reed's pointwise convergence criteria, Fund. Math. 86 (1974), 1-7. MR 50:4845

2. A. M. Bruckner, Differentiation of real functions, Lecture Notes in Math., no. 659, Springer Verlag, 1978. MR 80h:26002

3. A. M. Bruckner and J. G. Ceder, Darboux continuity, Jahresber. Deutsch. Math.-Verein. 67 (1965), 93-117. MR 32:4217

4. A. M. Bruckner, J. G. Ceder, and T. L. Pearson, On Darboux functions, Rev. Roumaine Math. Pures Appl. 19 (1974), no. 3, 977-988. MR 50:4846

5. A. M. Bruckner and J. L. Leonard, Stationary sets and determining sets for certain classes of Darboux functions, Proc. Amer. Math. Soc. 16 (1965), 935-940. MR 32:1303 
6. J. G. Ceder, Differentiable roads for real functions, Fund. Math. 65 (1969), 351-358. MR 40:4398

7. J. G. Ceder and T. L. Pearson, Insertion of open functions, Duke Math. J. 35 (1968), 277-288. MR 36:6556

8. _ A survey of Darboux Baire 1 functions, Real Anal. Exchange 9 (1983-84), no. 1, 179-194. MR 86a:26003

9. K. Ciesielski and A. W. Miller, Cardinal invariants concerning functions whose sum is almost continuous, Real Anal. Exchange 20 (1994-95), no. 2, 657-672. MR 96h:26003

10. Z. Grande, On the Darboux property of the sum of cliquish functions, Real Anal. Exchange 17 (1991-92), no. 2, 571-576. MR 93f:26001

11. A. Maliszewski, Sums of bounded Darboux functions, Real Anal. Exchange 20 (1994-95), no. 2, 673-680. MR 96f:26002

12. - On theorems of $P u \&$ \& $P u$ and Grande, Math. Bohemica 121 (1996), no. 1, 83-87. MR 97c:26006

13. I. Maximoff, Sur les fonctions ayant la propriété de Darboux, Prace Mat.-Fiz. 43 (1936), $241-265$.

14. Sur la transformation continue de fonctions, Bull. Soc. Phys. Math. Kazan 12 (1940), 9-41. MR 7:420g

15. - On functions of class 1 having the property of Darboux, Amer. J. Math. 65 (1943), 161-170. MR 4:213b

16. R. J. O'Malley, Baire* 1, Darboux functions, Proc. Amer. Math. Soc. 60 (1976), 187-192. MR 54:5405

17. D. Preiss, Maximoff's theorem, Real Anal. Exchange 5 (1979-80), no. 1, 92-104. MR 80m:26007

18. J. Smítal, On approximation of Baire functions by Darboux functions, Czechoslovak Math. J. 21 (1971), no. 96, 418-423. MR 45:470

19. J. Stallings, Fixed point theorem for connectivity maps, Fund. Math. 47 (1959), 249-263. MR 22:8485

20. J. Young, A theorem in the theory of functions of a real variable, Rend. Circ. Mat. Palermo 24 (1907), 187-192.

21. Z. Zahorski, Sur la première dérivée, Trans. Amer. Math. Soc. 69 (1950), 1-54. MR 12:247c

Department of Mathematics, Pedagogical University, Pl. Weyssenhoffa 11, 85-042 Bydgoszcz, Poland

E-mail address: amal@wsp.bydgoszcz.pl 